\title{
Las representaciones de glotón (Gulo gulo L.) en el arte paleolítico pirenaico y un nuevo hallazgo de arte mueble en el alto valle del Jarama (Guadalajara) *
}

\author{
Jesús F. JORDá PARDO ** \\ Miguel Angel Garcia Valero $* \star *$
}

\section{EL GLOTÓN (GULO GULO L.): SISTEMÁTICA, MORFOLOGIA, COSTUMBRES, HÁBITAT Y DISTRIBUCIÓN}

El glotón (Gulo gulo L., 1758) pertenece al Orden Carnivora y ocupa un lugar dentro de la Familia Mustelidae, siendo la especie gulo la única integrante del género Gulo, caracterizándose por ser la forma de mayor tamaño dentro de esta Familia. Para su clasificación sistemática y su caracterización morfológica y ecológica hemos utilizado las obras de Burton (1978) y de Schilling, Singer y Diller (1987), que seguiremos en estos aspectos a lo largo de este trabajo.

Morfológicamente es algo mayor que un zorro, parecido a un tejón, pudiendo asimilarse a un hibrido de marta y oso, diferenciándose de este último por presentar una cola peluda bien visible, un cuerpo compacto, \footnotetext{
1988 ".

* Trabajo presentado al "Congreso Internacional de Historia de los Pirineos, Cervera,

** Instituto Tecnológico Geo-Minero de España.

*** Museo Provincial de Guadalajara.
} 

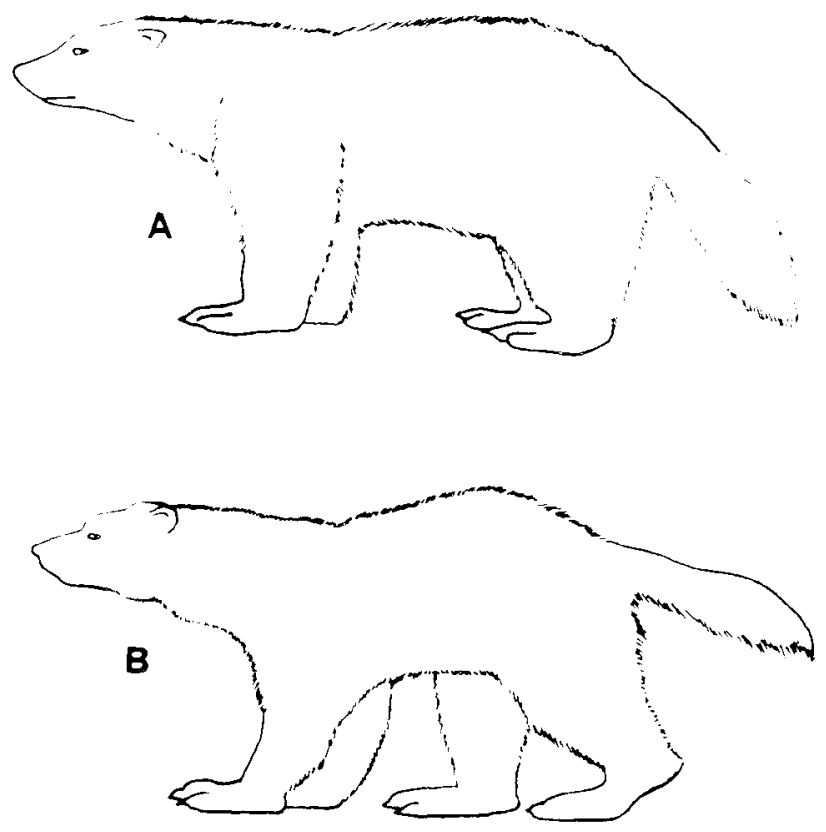

Fig. 1. Perfil de glotón actual: A) estático, B) en movimiento.

patas relativamente cortas y robustas, anchos pies acabados en una poderosa zarpa armada de largas y afiladas uñas, pelaje espeso, ojos y orejas relativamente pequeños, sin sobresalir aquellas del perfil de la cabeza, hocico bien marcado y mandibula y dentadura potentes. El pelaje, lanudo y denso, es de color pardo oscuro, con una banda en la frente y en los flancos, de color blanco amarillento a gris (fig. 1).

Las medidas corporales son las siguientes: longitud de la cabeza y el cuerpo, $70-90 \mathrm{~cm}$; longitud de la cola, $12-30 \mathrm{~cm}$; altura hasta la cruz, $35-45 \mathrm{~cm}$, peso corporal, $9-20 \mathrm{~kg}$, ocasionalmente hasta $30 \mathrm{~kg}$. Existe un claro dimortismo sexual, siendo el macho menor y menos pesado que la hembra.

En cuanto a sus hábitos, es un animal solitario, principalmente nocturno, y posiblemente tiene ritmos de 3-4 horas de actividad y descanso. Vive en familia de carácter matriarcal y el apareamiento tiene lugar en otoño, aunque probablemente se retrasa la implantación dado que la cría no nace hasta febrero o marzo e incluso mayo (Burton, 1978), si bien otros autores (Schilling, Singer y Diller, 1987) lo situan en primavera o verano (abril - agosto). La gestación dura de 8 a 9 meses, naciendo 
normalmente de 2 a 5 crías, que son amamantadas durante unas 10 semanas. Permanecen junto a la madre entre uno y dos años, según los autores citados, alcanzando la madurez sexual a los 3-4 años. La madre los conduce lejos para que encuentren sus propios territorios. Su esperanza de vida es aproximadamente de 15 años.

Es un animal que caza en solitario o en parejas en época de apareamiento, principalmente de noche, y con poca destreza para cazar al acecho, si bien corre con velocidad moderada y gran resistencia, siendo buen nadador. Ocasionalmente, aleja a otros depredadores de las piezas que ha capturado, pudiendo alimentarse de carroña, gracias a sus fuertes dientes y poderosas mandibulas, con las que tritura huesos grandes. Por lo general come más de los que necesita, de ahi su nombre, si bien su estómago es grande. Su alimentación es principalmente animal: carroña, micromamíferos como el lemming, grandes mamiferos, como corzos, ovejas, renos y alces, aves que anidan en el suelo y sus huevos y polluelos, anfibios, peces, insectos y moluscos. Excepcionalmente incluye en su alimentación frutos y bayas. Emite diversos sonidos, como gruñidos, silbidos y siseos en caso de hambre, temor, o agresividad, o sollozos y chillidos durante los juegos.

No construye su propio hábitat, utilizando cualquier refugio disponible, incluyendo los hechos por el hombre. Generalmente se trata de cavidades entre las rocas o debajo de las raices, poseyendo varios refugios en su territorio, que utiliza para protegerse y descansar en sus correrias. Las crias nacen en oquedades de árboles, rocas e incluso galerias en la nieve. Excepcionalmente trepan a los árboles pero, por lo general, permanecen en el suelo.

Su territorio abarca entre 500 y $2000 \mathrm{~km} 120$ y en el territorio más extenso de un macho, pueden vivir hasta tres hembras en sus propios territorios. Entre sus pocos enemigos naturales se encuentran el lobo, el oso pardo y el lince, si bien el hombre es su mayor perseguidor, ya que este animal es destructivo y tiene fama de cazador de renos. Actualmente los esquimales lo cazan para aprovechar su piel. Con todo, aunque su número es cada vez más reducido debido a las persecuciones, todavia es frecuente en algunas áreas.

Los bosques perennifolios fríos (paisaje de taiga), de 250 a $4000 \mathrm{~m}$ sobre el nivel del mar, suele ser su ecosistema caracteristico, si bien en verano los glotones que viven más al $\mathrm{N}$, sustituyen los bosques y matorrales por la tundra, así como zonas rocosas y de turba. Su distribución se extiende por regiones de la tundra y la taiga de Eurasia y Norteamérica, desde Escandinavia y $\mathrm{N}$ de Leningrado hasta Kamtchaka, Alaska y 
Canadá por el $\mathrm{S}$ hasta Mongolia septentrional, hasta Sajalín y hasta los estados septentrionales de USA.

Las condiciones climáticas del hábitat actual del glotón son (Hork, 1951): precipitaciones del orden de los 200 a los $700 \mathrm{~mm}$ anuales, temperaturas medias en enero entre $-70^{\circ}$ y $-10^{\circ} \mathrm{C}$, temperaturas medias en julio entre $0^{\circ}$ y $20^{\circ} \mathrm{C}$, entre 25 y 150 dias al año con una temperatura por encima de los $10^{\circ} \mathrm{C}$ y entre 0 y 160 días al año con una temperatura por encima de los $5^{\circ} \mathrm{C}$.

Más datos acerca de las características del glotón aparecen recopilados en el extenso trabajo de I. Barandiarán (1974).

\section{EL GLOTÓN (GULO GULO L.) EL EN ÁMBITO DEL ARTE PALEOLÍTICO PIRENAICO}

Durante el Cuaternario existieron en Europa varias especies de glotones que se sucedieron en el tiempo. Asi, en el Pleistoceno Medio, a la especie Gulo schlosseri Kormos les sucede G. gulo, lo cual se documenta en Mosbach y Chu-ku-Tien (Kurten, 1968). Sin embargo, no será hasta el Riss y el Würm, en Europa, cuando aparece asociado a conjuntos faunísticos claramente indicadores de climas fríos. Con todo, sus restos no son abundantes en yacimientos prehistóricos, pese a que en el Paleolítico Superior europeo pudo ser relativamente frecuente (Kurten, 1968).

La extensión meridional del glotón pleistoceno se asocia a la del reno, documentándose en yacimientos pirenaicos como la Cueva de L'Herm (Ariège, Francia) (Barandiarán, 1974) y la de Lezetxiki (Mondragón, Guipúzcoa), donde Altuna $(1963,1972)$ exhumó un fragmento de maxilar superior izquierdo en un contexto Gravetiense.

La aparición de restos de reno en la franja pirenaica y subpirenaica (Altuna, 1971), especie cuya dispersión geográfica y ecosistemas son paralelizables a los del glotón, nos inducen a considerar que el ámbito pirenaico fue durante el Pleistoceno Superior un lugar donde este mustélido pudo desarrollarse dentro de un biotopo adecuado. El registro fósil nos confirma dicha hipótesis.

Así mismo, el Arte Paleolítico reafirma la existencia del glotón en esta zona, documentándose representaciones parietales y muebles en 

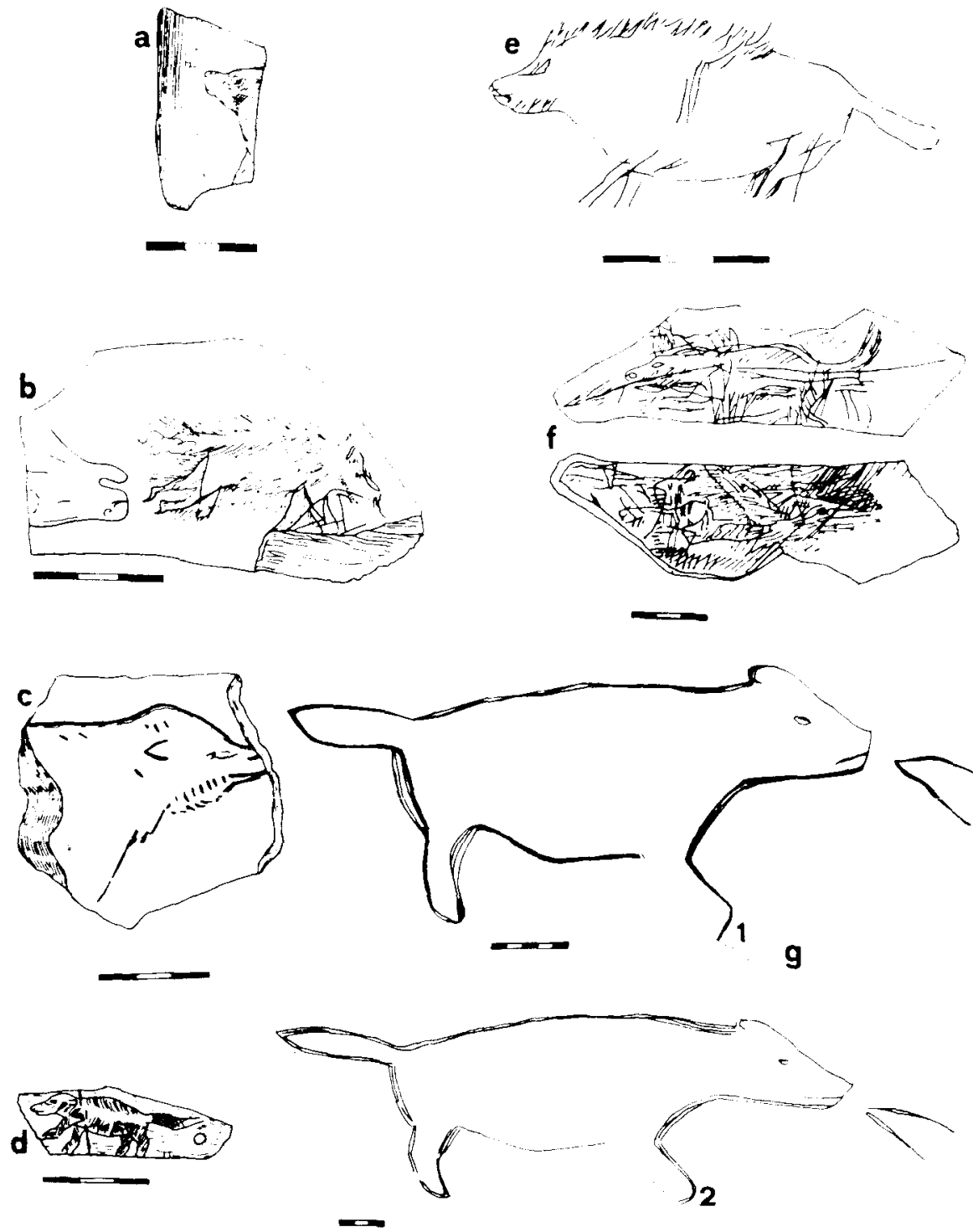

Fig. 2. Representaciones de glotones en el Arte Paleolitico occidental: a) Isturiztz (según R. de Saint Périer, 1936: dibujo de J. Bouyssonie); b) Saulges o Lorthet (según Breuil, 1937); c) Lorthet (calco de I. Barandiarán); d) Les Eyzies (según Capitán, Breuil y Peyrony); e) Laugerie Haute (dibujo sobre fotografía de Roussot, 1965); f) Trois Frères (según L. Bégouen, 1939: dibujo de J. Bouyssonie); g) Los Casares (g.1, calco directo de I. Barandiarán, g.2, calco sobre la fotografia de 1. Barandiarán). 
Las representaciones de glotón (Gulo gulo L.) en el arte paleolítico...

una serie de cuevas (Barandiarán, 1974) que a continuación exponemos, agrupándolas en tres zonas: Pirenaica, Norpirenaica y Surpirenaica (fig. 2).

\section{Zona Pirenaica:}

- Isturitz (Pirineos Atlánticos): En los niveles del Magdaleniense medio se localizó una pieza de arte mueble, elaborada sobre un fragmento óseo, que representa un posible glotón. Esta pieza presenta grabada la parte anterior de la cabeza de un carnivoro. Su atribución a esta especie viene dada por las características morfológicas de su hocico, pelaje espeso y frente abombada (Saint Perier, 1936; Barandiarán, 1974) (fig. 2.a).

- Mas D'Azil (Ariege): Una dudosa representación de este mustélido, aparece grabada sobre una placa de arenisca (Chollot, 1964). Su pertenencia a este yacimiento es problemática, ya que mientras Capitán, Breuil y Peyrony (1910) la citan como procedente de Gourdan, Barandiarán (1974) se inclina por ubicarla en Mas D'Azil, y contextualizarla dentro del Magdaleniense Superior.

- Trois Frères (Ariège): Procedente de este yacimiento existe una placa de arenisca sobre la que se han grabado en ambas caras diversas incisiones y dos figuras. Una de ellas corresponde a un mustélido, encajando perfectamente con la morfologia de Gulo gulo (Barandiarán, 1974). Es de destacar la interpretación que dieron Bègouen y Koby (1951) a esta figura, en la cual el glotón aparece atravesado por un venablo, e incluso hacen corresponder la cabeza grabada en la otra cara de la pieza con un glotón de fauces abiertas (fig. 2.f).

- Saulges o Lorthet (Pirineos): Sin procedencia ni contexto definidos (Barandiarán, 1974), existe una placa de esquisto, en la que en una de sus caras se representa una figura completa de glotón (Capitán, Breuil y Peyrony, 1924). Breuil (1937) destaca como elemento clave a la hora de clasificar esta representación la terminación en zarpas ganchudas de las extremidades posteriores. Una peculiaridad de esta figura que resaltó Obermaier (1940) se refiere a la existencia de dos dibujos superpuestos, uno que representa el animal en movimiento, de perfil, y otro que es una visión de su piel extendida (fig. 2.b).

- Lorthet (Pirineos): Sobre una placa de arenisca destaca, entre otras representaciones grabadas, la de un glotón, definida por la cabeza y la parte delantera del animal, con trazo bien elaborado, atribuible al Magdaleniense Superior (Barandiarán, 1974). Esta representación ha sido una de las mas aceptadas por la comunidad científica: Capitán, Breuil y Peyrony (1910), Begouen y Koby (1951), Koby (1960) y Chollot (1964). 
Las características morfológicas que presenta esta imagen encajan perfectamente con las descritas en el apartado anterior (fig. 2.c).

Otro posible grabado de este animal se atribuye a esta localidad (Barandiarán, 1974). Breuil (1937) en función de la forma de detallar las zarpas, semejantes a las de la figura de Saulges o Lorthet, identificó dicha representación como de la especie que nos ocupa.

Zona Norpirenaica:

- Les Eyzies (Dordogne): Con probabilidad, a esta cueva pertenece una de las mejores representaciones de glotón en el arte paleolítico. El soporte consiste en un colgante de cuerno de reno, y en él aparece grabado una figura que Breuil (Breuil, Capitán y Peyrony, 1910) consideró como la mas acertada imagen de los rasgos del glotón (largo pelaje y ancha cola). En el caso de que provenga de este yacimiento, correspondería al Magdaleniense Medio, o más reciente (Barandiarán, 1974) (fig. 2.d).

Laugerie-Haute (Dordogne): Sobre un fragmento de cuerno de reno aparece en este yacimiento una figura grabada en los estratos correspondientes al Magdaleniense Inferior. Su atribución es muy discutida, no obstante Barandiarán (1974) siguiendo los criterios de Lalanne (1909), considera que puede corresponder a este mustélido, especialmente por su cabeza y tronco macizos y la longitud de su cola (fig. 2.e).

\section{Zona Surpirenaica:}

- Los Casares (Guadalajara): En este importante santuario rupestre paleolítico Cabré (1934) localizó la única figura grabada de glotón que se conoce sobre soporte parietal, con unas características morfológicas perfectamente ajustables a las de este carnivoro, como son un cuerpo macizo, cabeza destacada con pequeñas orejas que apenas sobresalen, extremidades cortas y anchas, y cola mediana, si bien la gran cantidad de pelaje que tiene este animal no aparece representada en esta figura. Este hecho, según Barandiarán (1974), es relevante, sobre todo, si tenemos en cuenta que los autores de estos grabados reflejaron, con gran precisión, en otras representaciones de la misma cueva una espesa capa de pelaje. Cronológicamente esta figura es atribuida por Beltrán (1968), en función de la técnica de grabado, a una etapa comprendida entre el Solutrense antiguo y el Magdaleniense IV, mientras que para Jordá (1986) se situaria entre el Solutrense y el Magdaleniense Superior (fig. 2.g). 


\section{UN NUEVO HALLAZGO DE ARTE MUEBLE EN LA CUEVA DE JARAMA II (VALDESOTOS, GUADALAJARA)}

\subsection{Localización, circunstancias del hallazgo y problemática de estudio}

La Cueva de Jarama II esta situada en la margen izquierda del río Jarama, en el término municipal de Valdesotos (provincia de Guadalajara), a $940 \mathrm{~m}$ de altitud sobre el nivel del mar, dentro de la hoja $n .{ }^{\circ} 485$ del mapa Topográfico Nacional a E. 1:50.000. Es una cueva de escasas dimensiones cuya situación topográfica es bastante compleja, por lo que el acceso a ella es dificil y peligroso. La entrada se encuentra en la pared vertical del acantilado o farallón que forma en ese punto la margen izquierda del rio, a unos $5 \mathrm{~m}$ por debajo de la superficie topográfica superior, en una pequeña cornisa situada a $22 \mathrm{~m}$ del suelo en caida vertical. La cueva, orientada al $\mathrm{N}, \mathrm{y}$ de dirección NS, en un estrecho conducto o galeria de $10 \mathrm{~m}$ de recorrido visible, con una anchura máxima de $4 \mathrm{~m}$ y mínima de $1 \mathrm{~m}$, oscilando su altura entre 2,5 y $0,5 \mathrm{~m}$. El suelo de esta galería es practicamente horizontal, con una ligera inclinación hacia el interior, y esta formado en su totalidad por sedimentos que contienen restos arqueológicos del Paleolítico Superior y del Bronce Antiguo.

La pieza que presentamos fue extraida irregularmente del contexto arqueológico con anterioridad a enero de 1983, fecha en que fue dada a conocer al Prof. Dr. F. Jordá Cerdá. A partir de este momento, un equipo * dirigido por este investigador comenzó los estudios sistemáticos en dicha cavidad, realizándose varias prospecciones en la zona. Desde 1985 y hasta la actualidad uno de los firmantes (J.F.J.P.) dirige el proyecto «Investigaciones Prehistóricas en el Alto Valle del Jarama (Guadalajara)" dentro del cual se ha llevado a cabo la excavación sistemática de este yacimiento, que ha aportado numerosa información acerca del contexto en que se encontró la mencionada representación mueble.

Debido a las especiales circunstancias que rodean la propiedad de la pieza, actualmente en depósito por su propietario en el Museo Arqueológico Nacional, únicamente pudimos acceder a ella en un corto espacio de tiempo en febrero de 1983, en el Laboratorio del Departamento de Prehistoria y Arqueología de la Universidad de Salamanca, gracias a la

* El equipo estuvo integrado por J. E. Aura Tortosa, F. J. González-Tablas Sastre, J. Bécares Pérez y J. F. Jordá Pardo. 
amabilidad de su dueño. Así, únicamente podemos contar con la documentación que obtuvimos en aquel momento, que se reduce a dibujos, diapositivas y numerosas fotografias en $b / n$ y color.

\subsection{Descripción de la pieza}

Se trata de una estatuilla de bulto redondo realizada en marfil, probablemente perteneciente a la defensa de un proboscideo, dado el tamaño de la pieza. El soporte presenta una serie de aiteraciones consistentes en: exfoliación paralela al eje longitudinal de la pieza oblicua a las superficies de los flancos, carbonataciones parciales, y fracturación reciente en orejas, patas y cola (fig. 3,4).

Las dimensiones de la pieza son:

- Longitud total ................................ 86,6 mm

- Anchura torácica ............................... 28,8 mm

- Anchura pelviana ............................... 22,3 mm

- Longitud de la cabeza ........................... 23,3 mm

- Anchura de la cabeza ......................... 10,6 mm

- Longitud de la oreja ............................ 6,6 mm

- Diámetro ocular ............................... 2,0 mm

- Longitud de la cola .............................. 23,3 mm

- Anchura de la cola ............................. 7,3 mm

- Altura hasta la cruz ............................. 32,0 mm

- Longitud extremidad delantera ..................... $8,0 \mathrm{~mm}$

- Longitud extremidad trasera ........................ 6,6 mm

- Anchura máxima extremidad anterior .............. 7,6 mm

- Anchura mínima extemidad anterior ................ 7,3 mm

- Anchura máxima extremidad posterior ..............10,0 mm

- Anchura minima extremidad posterior ............... $7,6 \mathrm{~mm}$

Las seis últimas medidas deben considerarse con reservas dada la fracturación a la que antes hemos aludido. No hemos podido obtener datos del espesor y peso de la pieza, dadas las circunstancias de su documentación.

La figura está representada de perfil, por lo tanto tenemos dos planos jerarquizados, anverso (el animal mira hacia la izquierda) y reverso.

Observando la figura en su conjunto, se advierte un cuerpo macizo, alargado, mas ancho hacia el torax, con un cuello muy corto, apenas delimitado, pecho amplio, extremidades cortas y poco robustas, cola larga 
y ancha, y cabeza almendrada. Esta última presenta en su extremo posterior unas orejas pequeñas y de contorno trapezoidal, así como unas incisiones puntuales a ambos lados que indican la posición de los ojos. El hocico, la boca y las fosas nasales no aparecen marcadas. Hay que tener en cuenta que tanto las extremidades como la cola aparecen fracturadas por lo que no se conserva su longitud original.

El contorno de la figura viene definido por fomas suaves y redondeadas, si bien las líneas cervico dorsal y ventral son marcadamente rectilíneas guardando cierta convexidad, que confieren un aspecto estático al animal. La mitad inferior de la pieza presenta una mayor angulosidad, definida por las intersecciones del pecho con la cabeza y extremidades anteriores, el vientre y ambas extremidades, y las extremidades posteriores y la cola. Resaltaremos que en el anverso la cola aparece menos marcada que el reverso, aunque presenta la línea del despiece sugerida por una profunda incisión.

En el anverso la figura se percibe mas completa, ya que únicamente ha perdido un fragmento de la oreja que no ha perjudicado su perfil, y en la cola presenta un escamamiento en su zona media. La exfoliación es mas apreciable en esta cara, observándose hasta 5 planos y una especie de nudosidad en la zona cervical. Las concreciones carbonatadas son mas abundantes en las zonas inferiores, afectando al maxilar inferior, pecho y vientre.

El reverso esta mas deteriorado, con ausencia total de oreja, y marcada fracturación de ambas extremidades y cola. Los planos de exfoliación aparecen menos marcados, apreciándose sólo la continuación de los dos mas profundos del anverso. Las concreciones se presentan fundamentalmente en la parte anterior.

\subsection{Atribución de la pieza a la especie Gulo gulo L}

A primera vista, la silueta de la figura que nos ocupa es similar a las otras representaciones de glotón anteriormente descritas, especialmente a la de Los Casares (fig. 4). Por tanto, y una vez comparada dicha silueta con las de otras especies de carnivoros actuales, especialmente mustélidos, considerados posible su adscripción a la especie Gulo gulo L, lo cual corroboramos mediante el estudio de las proporciones y medidas.

Para atribuir esta figura a la especie mencionada nos hemos basado en las proporciones reales del animal, comparándolas con las de la pieza 

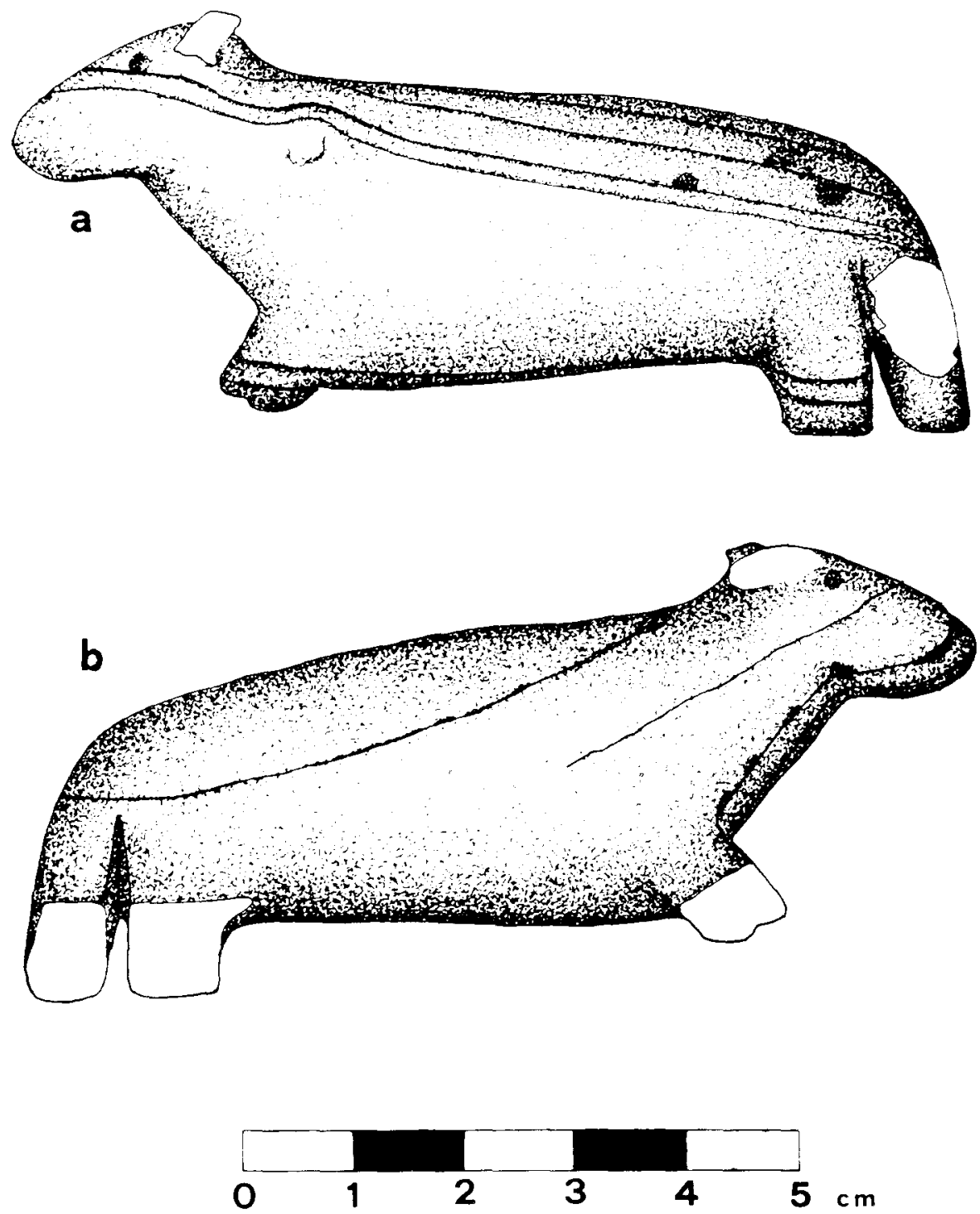

Fig. 3. Representación de glotón en bulto redondo de Jarama II, a) anverso, b) reverso (calco sobre fotografía de $M$. A. García Valero). 


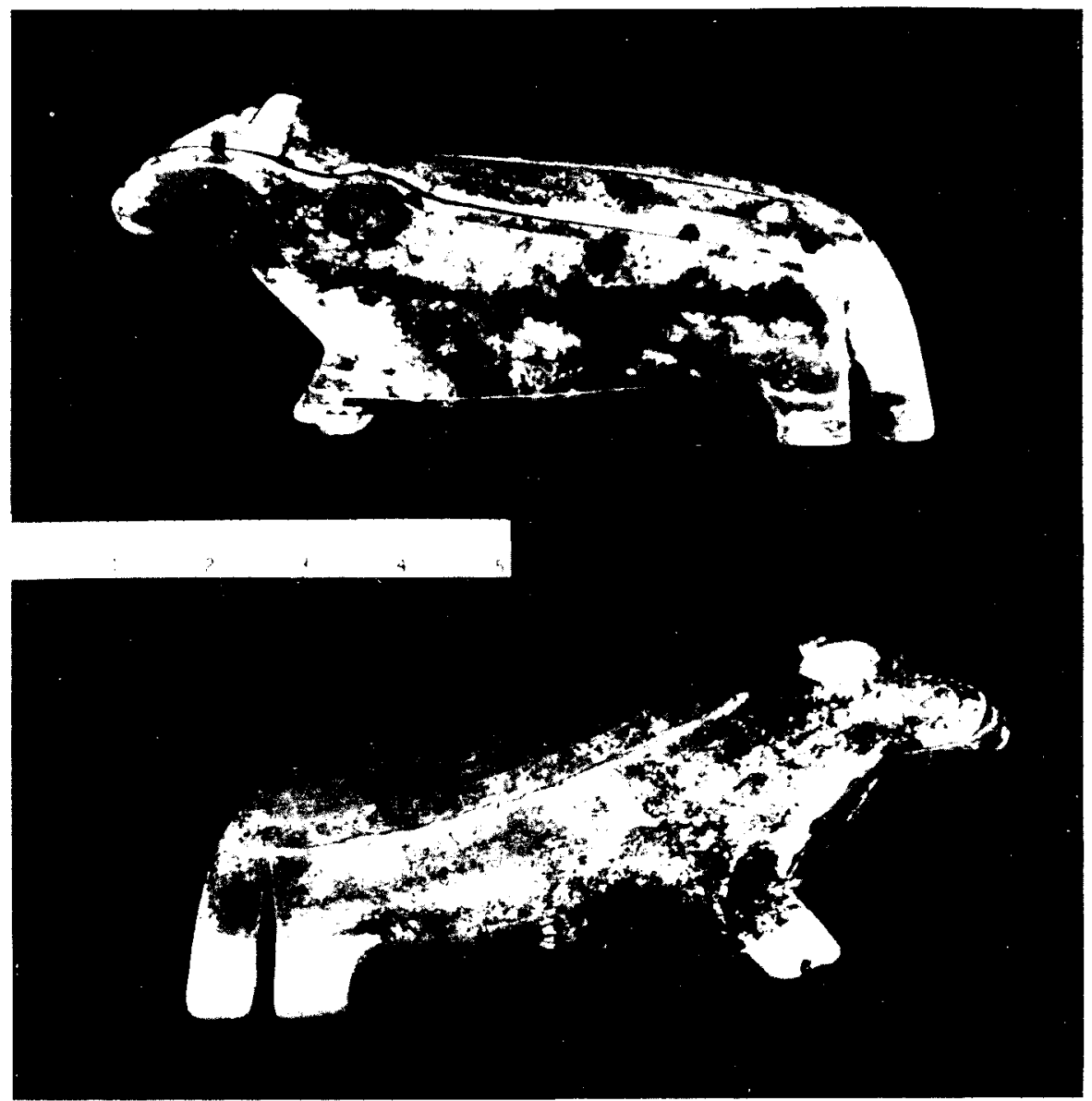

Fig. 4. Fotografia de la figura de Jarama II (foto J. F. Jordá).

que nos ocupa. En el siguiente cuadro confrontamos las dimensiones de la especie actual y las de la de Jarama II:

Longitud cabeza-cuerpo:

Altura hasta la cruz:

Longitud cola:

$\begin{array}{cc}\text { especie actual } & \text { figura Jarama } \\ 700-900 \mathrm{~mm} & 86,6 \mathrm{~mm} \\ 350-450 \mathrm{~mm} & 32,0 \mathrm{~mm} \\ 120-300 \mathrm{~mm} & 23,3 \mathrm{~mm}\end{array}$

Comparando las medidas del glotón actual y de la figura en estudio, observamos que existe una clara correspondencia entre los tres parámetros considerados, resaltándo que si multiplicamos por 10 las medidas 
de la figura de Jarama II, obtenemos unas cifras muy similares a las de la especie actual.

Como se deduce del cuadro anterior, la relación entre la longitud cabeza-cola y la altura hasta la cruz es de 2 a 1 para la especie actual. Dado que las extremidades de la pieza en estudio se hallan fragmentadas en sus extremos podemos considerar que la altura hasta la cruz de esta sería del orden de 43,3 mm, guardando así la proporción antes indicada. Siguiendo estos argumentos, la longitud de las extremidades se incrementaría en unos $11 \mathrm{~mm}$. Por tanto, la pieza en su estado original, seria bastante mas esbelta de lo que nos resta de ella en la actualidad.

Por todo lo expuesto anteriormente, creemos que no resulta aventurado atribuir esta figura de bulto redondo a la especie de mustélido aqui tratada, Gulo gulo L (glotón).

\subsection{Cronologia}

Como dijimos anteriormente, la estatuilla apareció fuera de contexto estratigráfico claro. No obstante, las excavaciones arqueológicas que hemos efectuado en el citado yacimiento, nos han permitido documentar un horizonte correspondiente a un momento del Paleolítico Superior, que presumiblemente se trataria del Magdaleniense Inferior.

El argumento que utilizamos para determinar esta cronologia es una azagaya en asta de sección cuadrangular con incisiones longitudinales y transversales, con claros paralelos formales en el ámbito pirenaico y cornisa cantábrica. Con similar cronologia destacan las azagayas encontradas en el nivel VII de Ekain (Guipúzcoa) (Baldeón, 1984), en el nivel 8 del Castillo (Cantabria) (Cabrera Valdés, 1984), en el nivel 4 de Rascaño (Cantabria) (González Echegaray y Barandiarán Maestu, 1981), en los grupos II y III del Juyo (Cantabria) y nivel 4 del Cierro (Asturias) (Utrilla, 1981), en Balmori y en el nivel D de Cueto de la Mina (Asturias) (Vega del Sella, 1916, 1930).

\section{RELACIONES Y CONTEXTUALIZACIÓN DEL NUEVO HALLAZGO EN EL ARTE PALEOLITICO PIRENAICO}

Comparando la estatuilla estudiada con las representaciones muebles y parietales de esta especie en el Arte Paleolítico del ámbito pire- 
naico, podemos afirmar que existen ciertos parecidos formales entre la pieza de Jarama II y las figuras pertenecientes a los yacimientos citados en el epigrafe 2.

El contorno del cuerpo tiene una gran similitud con todas las siluetas (fig. 5) si bien con la que guarda un mayor parecido es con la de Los Casares. No obstante, todas muestran cierto dinamismo, reflejado por la actitud de las extremidades y de la cola, mientras que en nuestro caso estas indican una menor movilidad e incluso estatismo. En la mayoría de las representaciones aparecen las cuatro extremidades, siendo la de Los Casares y la que nos ocupa las únicas que muestran unicamente dos, tratándose de un convencionalismo clásico en el Arte Paleolítico a la hora de representar los animales de perfil. La cola en la totalidad de los casos, aparece enhiesta, mientras que en nuestro ejemplar se encuentra pegada a los cuartos traseros.

El pecho rectilíneo y manteniendo un ángulo casi recto con la extremidad delantera es una característica que encontramos tanto en Los Casares como en Jarama II. En estos dos yacimientos resaltaremos que las extremidades mantienen una misma actitud, apareciendo las delanteras extendidas hacia delante, mientras que las traseras están verticales.

La morfología de la cabeza guarda ciertas similitudes con las de Les Eyzies, Isturitz y Los Casares, apareciendo las orejas sobresaliendo del contorno en los ejemplos de Los Casares y Jarama II. El hocico se denota en la práctica totalidad de las representaciones, exceptuando la de Los Casares y Jarama II, mientras que la línea de la boca esta ausente tanto en nuestra figura como en las de Saulges o Lorthet y la de Isturitz.

El pelaje es un rasgo anatómico que aparece en las figuras de Saulges o Lorthet, Laugerie-Haute, Les Eyzies, Lorthet, Isturiz y Trois Frères, siendo las representaciones de Jarama II y Los Casares, las únicas que no presentan estas características.

Las representaciones conocidas de glotones se localizan fundamentalmente, como ya hemos visto, en la franja pirenaica, lo cual se ajusta a los requisitos climáticos necesarios para la supervivencia de este animal. La mayoria de las figuras corresponden al Magdaleniense, período en el que el clima en la zona pirenaica sería extremadamente frio. Un hecho relevante es que algunas de las figuras aparecen grabadas sobre restos óseos de reno, especie que va intimamente unida a un ambiente extremadamente frio, y por lo tanto similar al del glotón.

En nuestro caso no contamos con datos climáticos que nos informen de la existencia de un clima tan frio como el existente en los Pirineos 


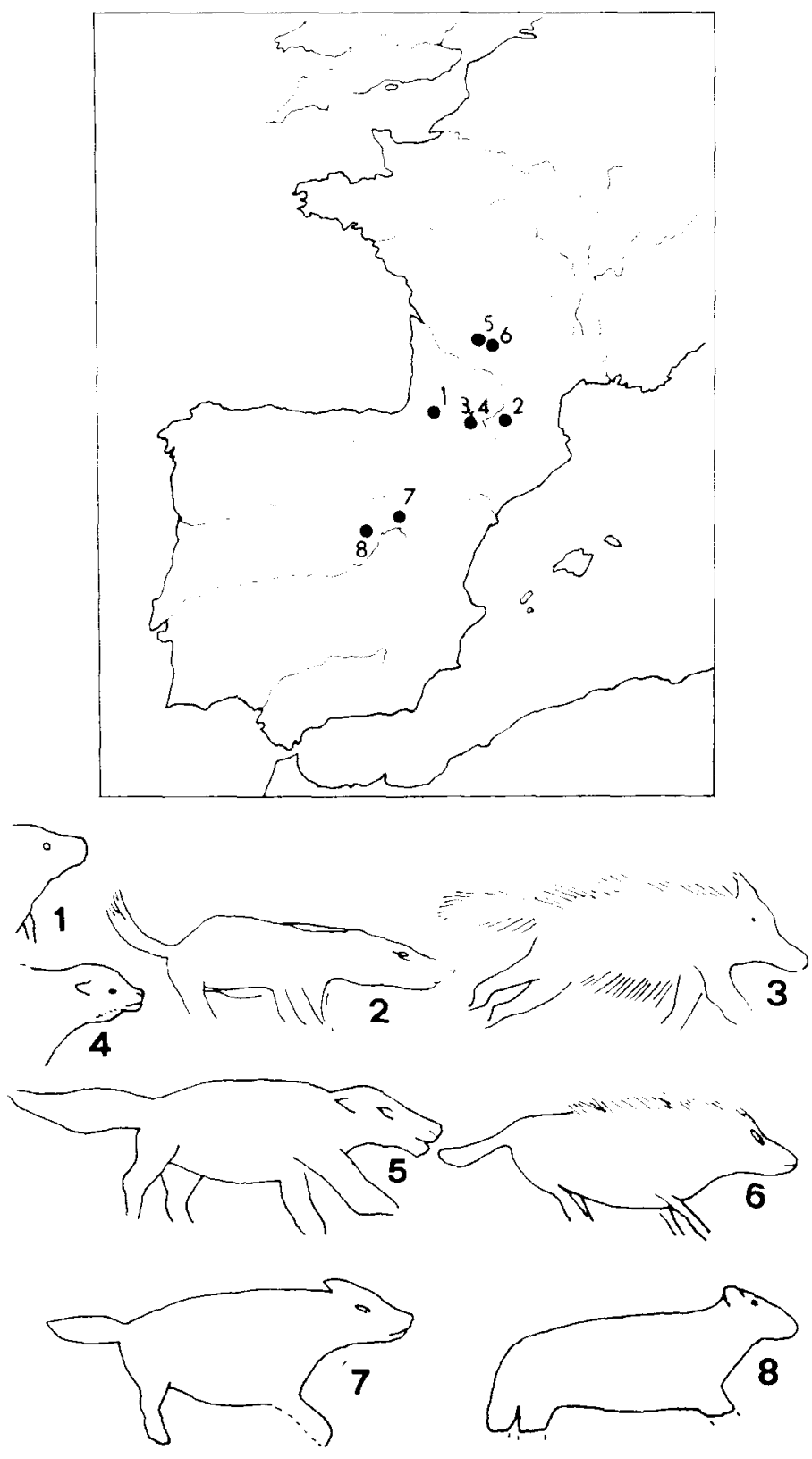

Fig. 5. Mapa de localización de las distintas representaciones de glotón y sus siluetas: 1) Isturitz, 2) Trois Frères, 3) Saulges o Lorthet, 4) Lorthet, 5) Les Eyzies, 6) Laugerie Haute, 7) Los Casares, 8) Jarama II. 
durante este periodo. No obstante, la adscripción de nuestra figura al Magdaleniense Inferior, y la posibilidad de que la de Los Casares pertenezca igualmente a esta etapa (Jordá Cerdá, com. pers.), nos inducen a suponer un clima suficientemente frío en la zona del $N$ de Guadalajara, que permitiría la presencia de esta especie. Todo ello contribuye a considerar la presencia de glotones al $\mathrm{S}$ de los Pirineos durante el Pleistoceno Superior final, que constituirian poblaciones aisladas en núcleos montañosos de extrema rigurosidad climática.

Puede parecer extraño que en un área relativamente al $\mathrm{S}$ de los Pirineos, aparezcan representaciones de este animal, pero debemos tener en cuenta que el clima debía ser al menos tan extremacio en esta zona que, por ejemplo, en la Cornisa Cantábrica (zona mas cercana a los Pirineos latitudinalmente), donde, hasta el momento no se han hallado restos óseos ni representaciones de esta especie, si bien contamos con manifestaciones artísticas y con restos óseos de reno, especie que como dijimos anteriormente coincide en el mismo biotopo con Gulo gulo. No se trataría de un hecho aislado si tenemos en cuenta la cercana representación de Los Casares, considerando que estas manifestaciones se basaban en una "visión que se formaba gracias al contacto con una fauna cuya variedad y riqueza, en nuestras latitudes, no ha vuelto a tener equivalentes" (Clottes, 1986-87).

Dentro del bestiario representado en el Arte Paleolítico Occidental, y concretamente en el Pirenaico, el tratamiento de este mustélidos es muy escaso, y en algunas ocasiones su atribución es dudosa. Por otra parte las esculturas de bulto son también escasas dentro del Arte Mueble (Garcia, 1987), si bien resaltaremos un magnífico ejemplo de caballo esculpido en marfil, hallado en la Grotte de Lourdes (Hautes Pyrénées) en un contexto magdaleniense, que constituye la más cercana manifestación en bulto redondo y en marfil al hallazgo de Jarama II (Clottes, 1976).

Por último, consideramos que la representación en bulto redondo de glotón, encontrada en Jarama II (Alto Valle del Jarama), constituye no sólo un ejemplo de excepción entre las manifestaciones artísticas existentes de este mustélido, sino también dentro del repertorio de Arte Mueble del Paleolítico Superior occidental. 


\section{BIBLIOGRAFIA}

ALtUnA, J., 1963: “Primer hallazgo de glotón (Gulo gulo L) en la Peninsula Ibérica", Munibe, 3-4, San Sebastián, pág. 128.

- 1972: «Fauna de mamíferos de los yacimientos prehistóricos de Guipúzcoa. Con Catálogo de los Mamíferos Cuaternarios del Cantábrico y del Pirineo Occidental», Munibe, 1-4, San Sebastián.

BALDEÓN, A., 1984: "Industria ósea de Ekain», en ALtUNA, J. y MERINo,

J. M., El yacimiento prehistórico de la Cueva de Ekain (Deba, Guipúzcoa), San Sebastián. Editorial Eusko Iraskuntza, pág. 189-209.

Barandiará, I. 1974: “El Glotón (Gulo Gulo L.) en el Arte Paleolítico", Zephyrvs, XXV, pág. 177-196.

Begoǘn, H. y KoBY, F. ed., 1951: "Le Crâne de Glouton de la Caverne des Trois-Frères (Ariège)", Préhistoire et Spéléologie Ariégeoises, V, Tarascon, pág. 49-68.

Beltran, A., 1968: "Notas sobre la técnica de los grabados de las cuevas de Los Casares y Altxerri", Simposio Internacional de Arte Rupestre (Barcelona 1966), pág. 21-24.

BreUIL, H., 1937: “De quelques oeuvres d'art magdaléniennes inédites ou peu connues", lpek, 11, Berlín, pág. 1-16.

Burton, M., 1978: Guia de los Mamiferos de España y de Europa, Ediciones Omega, S. A., Barcelona, 264 páginas.

Cabré, J., 1934: "Las cuevas de los Casares y de la Hoz", Archivo Español de Arte y Arqueologia, X, Madrid, pág. 225-254.

Cabrera Valdés, V., 1984: El yacimiento de ia Cueva de El Castillo (Puente Viesgo, Santander). Bibliotheca Praehistorica Hispana, vol. XXII, Madrid. 485 páginas.

Capitán, L., Breuil, H. y Peyrony, D., 1910: La Caverne de Font-deGaume aux Eyzies (Dordogne), Mónaco.

- 1924: Les Combarelles aux Eyzies (Dordogne), Mónaco. 
Chollot, M., 1964: Musée des Antiquités Nationales. Collection Piette. Art mobilier préhistorique, París.

ClotTES, J., 1976: "Les Civilisations du Paleolithique Superieur dans Les Pyrinnees", La Prehistoire Française, vol. I, 2, Paris, pág. 1214-1231.

- 1986-87: "La determinación de las representaciones humanas y animales en el arte rupestre paleolítico", Bajo Aragón Prehistoria, VIIVIII, Caspe, pág. 41-68.

GarciA, M. A., 1987: "La esculpture prehistorique. Pour une technologie", Pre-Actes, Colloque International d'Art Mobilier Paleolithique, pág. 301-310.

Gonzalez Echegaray, J. y Barandiaran Maestu, I., 1981: El Paleolítico Superior de la Cueva del Rascaño (Santander). Monografías del Centro de Investigación y Museo de Altamira, núm. 3, Santander, 359 páginas.

Honk, Z., 1951: "A Method of the Quantitative Determination of the Climate in the Quaternay Period by Means of Mammal Association", Sbornik of the Geological Survey of Czechoslovakia, XVIII, pág. 209219.

Jorda Cerdá, F., 1986: «Paleolítico», en Jordá Cerda, F., Pellicer Catalá, M., Acosta martinez, P. y Almagro-Gorbea, M., Historia de España 1, Prehistoria, Editorial Gredos, Madrid, pág. 7-150.

KoBY, F., Ed. 1960: "Sur l'extension maxime vers le Sud-Ouest de quelques représentants de la faune froide Würmienne", Mammalia pleistocaenica, I, Brno, pág. 101-114.

KuRTEN, B., 1968: Pleistocene Mammals of Europe, Londres.

LALANNE, G., 1909: "Glouton gravé sur bois de renne de l'époque magdalénienne", Actes de la Société Linnéenne de Bordeaux, 62, Burdeos.

Obermaier, H., 1940: "The Treatment of Skins and Clothing Problems during Quaternary Times", Research and Progress, VI, 4, Londres, págs. 150-156.

Saint PeRIER, R. DE 1936: La grotte d'Isturitz. II, Le Magdalénien de la Grande Salle, Paris.

Schilling, D., Singer, D. y Diller, H., 1987: Guía de los Mamiferos de Europa, Ediciones Omega, Barcelona, 294 páginas.

Utrilla Miranda, P., 1981: El Magdaleniense Inferior y medio en la costa cantábrica, Monografías del Centro de Investigación y Museo de Altamira, núm. 4, Santander, 335 páginas.

Vega del Sella, Conde De LA, 1916: El Paleolítico del Cueto de la Mina, Memorias de la Comisión de Investigaciones Paleontológicas y Prehistóricas, núm. 13, Madrid, 94 páginas. 
Las representaciones de glotón (Gulo gulo L.) en el arte paleolítico...

- 1930: Las Cuevas de la Riera y Balmori (Asturias), Memorias de la Comisión de Investigaciones Paleontológicas y Prehistóricas, núm. 38, Madrid, 116 páginas. 